\title{
A Neonate with Aplasia Cutis Congenita Type VI with Junctional Epidermolysis Bullosa: A Very Rare Condition
}

\author{
Deepak SHARMA ${ }^{1}$, Sachin LAMBA², Anshul MAHESHWARI ${ }^{2}$, Sweta SHASTRI ${ }^{3}$
}

${ }^{1}$ Pt B.d. Sharma, Pgims, Rohtak, Haryana, India.

${ }^{2}$ S.m.s Medical College, Jaipur, Rajasthan, India.

${ }^{3}$ Nkp Salve Medical College, Nagpur, Maharashtra, India.

\section{ABSTRACT}

This study aimed to describe a case of aplasia cutis congenita with epidermolysis bullosa, as only a few reports of Bart's syndrome exist in the medical literature. Aplasia cutis congenita (ACC) is a rare dermatological condition characterized by the absence of skin. It has no proven etiology and hence stills remains a mystery for the dermatologist of the modern world. The most common body part affected is the scalp. ACC with epidermolysis bullosa (Bart's syndrome) is a very rare variant of aplasia cutis.

Key words: Aplasia cutis congenita, Bart's syndrome, epidermolysis bullosa, neonate, type VII collagen (COL 7A1)

\section{INTRODUCTION}

Aplasia cutis congenita (ACC) is a very rare congenital dermatological disorder characterized by the localized absence of skin, dermal appendages, and, in few rare cases, subcutaneous tissues. It was first described in medical literature by Cordon in 1767. ACC can involve any part of the body, but in the majority of the cases (84\%), the defect is found in the scalp (1). It usually presents as a solitary lesion on the scalp and may be as large as $70 \%$ of that site (2). The etiopathogenesis is uncertain, and it is postulated to be the result of more than one disease process. Hereditary factors, teratogens, or reduced blood supply to the skin are thought to be involved, which needs further investigation $(3,4)$. Most of the cases reported have sporadic inheritance, but few cases are reported to have either autosomal dominant or autosomal recessive inheritance. Frieden has classified aplasia cutis into nine types (3). The aplasia cutis congenita type 6 (Bart's syndrome) is an uncommon type of ACC, which presents as a glistening, reddish color ulceration with extended, clearly defined borders over the back and medial aspect of the foot. Usually, it extends up to the shin with unilateral or bilateral involvement. The present case was in clinical accordance with these characteristics.

\section{CASE PRESENTATION}

A term male baby of nonconsanguineous parents, weighing $2.8 \mathrm{~kg}$, was brought on the third day of his birth to the dermatology department with complaints of an ulcerated lesion on the left leg, predominantly on the medial aspect, since birth. This was the first child of the parents with an uneventful normal vaginal delivery. Both parents were obviously sound and had no abnormalities of skin, skin appendages, or mucous membrane. Dermatological examination revealed a well-demarcated, irregular area of eroded skin extending from the upper side of the knee joint to the base of toe nails of size around $15 \mathrm{~cm} \times$ $6 \mathrm{~cm}$ in the largest dimension with no overlying bullae (Figure 1). The skin adjacent to this lesion was normal, and a clear dividing line was present between the normal and the pathologic skin. The physical examination showed no oral erosions and no obvious malformation of the other systems. Nail dystrophy and joint contracture were absent on examination. Clinically a diagnosis of Bart's syndrome was made. The infant was seen after 2 weeks in the outpatient department; it had developed new bullae around the old lesion and other sites with blister formation (Figure 2). The previous lesion was healing with scarring, but without milia formation. Skin punch biopsy was obtained from one such fresh blister, which showed 


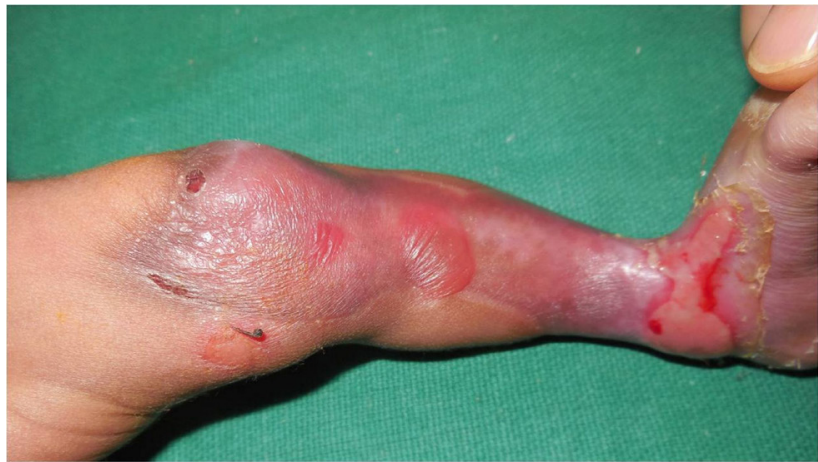

FIGURE 1: Aplasia cutis with epidermolysis bullosa on the leg of a 3-day-old neonate.

the separation between the epidermis and the dermis favoring a junctional variant of epidermolysis bullosa (Figure 3). The patient was then examined by the pediatrician for the presence of any associated congenital anomalies, but the examination was normal. The confirmation of the diagnosis was done with immunoelectron microscopy and direct immunofluorescence on skin biopsy using antibodies against the antigens of the basal membrane zone. The infant was prescribed the application of an emollient, and the parents were explained about the skin care and counseled for regular follow-up of the infant. The infant was lost to follow-up. The consent from the parents was obtained for the publication of the case report.

\section{DISCUSSION}

ACC is a rare dermatological anomaly characterized by the absence of skin over limited areas of the body at the time of birth. The reported incidence is one in 10,000 births. It usually happens

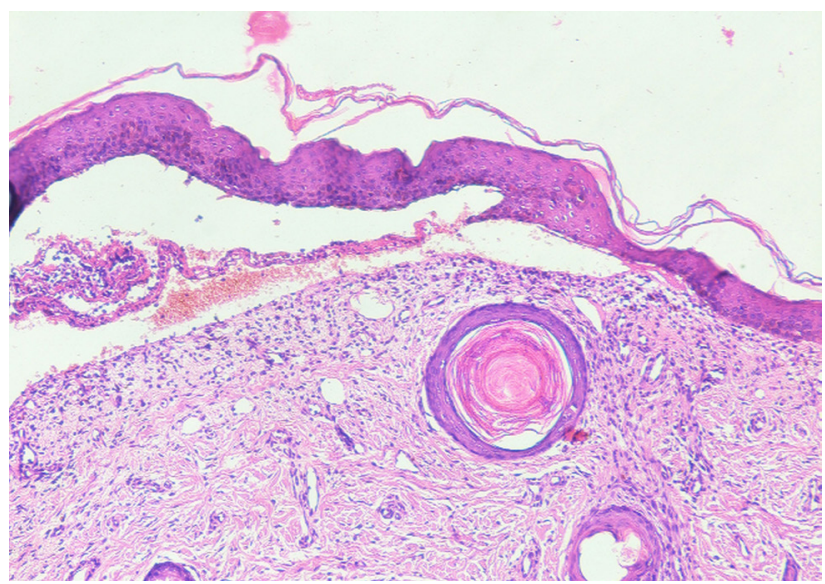

FIGURE 3: Histopathology showing clefting at the dermoepidermal junction (hematoxylin and eosin staining, 10× magnification).

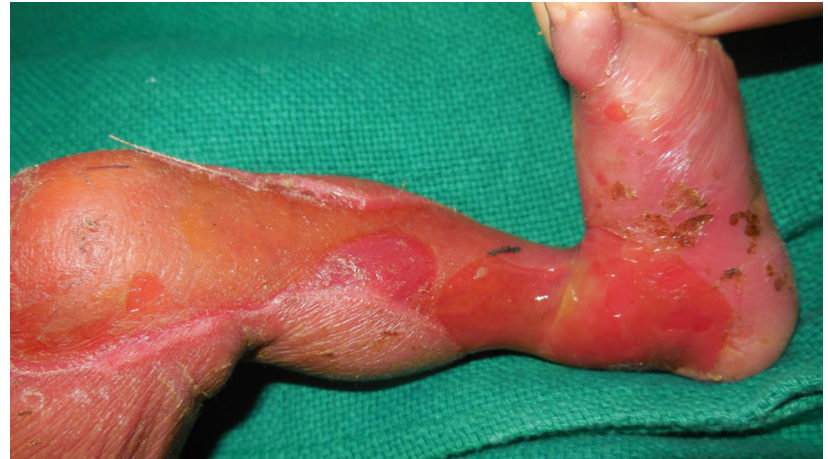

FIGURE 2: Eruption of new bullae at the age of 10 days.

sporadically with no history of sibling or parents involvement, although autosomal recessive and dominant inheritance have also been reported in medical literature.

Aplasia cutis type VI (also known as Bart's syndrome) is one the rarest form of aplasia cutis. It is usually associated with epidermolysis bullosa (EB). Clinical findings suggest that Bart's syndrome can be a part of any of the three subtypes of EB: epidermal, junctional, or dermal. It was first described in the literature by Bart in 1966 in a family with complete penetrance (5).

Later, Kanzler et al. (6) performed electron microscopy and immunofluorescence mapping of both regions of skin fragility and congenital localized absence of skin. Electron microscopy and immunofluorescence findings were identical at both the sites. These findings suggested that the area of congenital absence of skin had similar pathological changes as those seen in EB.

Zelickson et al., in their observational study on Bart's syndrome, showed that this entity had poorly developed anchoring fibrils. The ultrastructural analysis showed that the Bart's was the result of cleavage below the lamina densa. The genetic analysis suggested the location of the encoding gene on chromosome $3 p$, which was near the site of the gene encoding for type VII collagen (COL7A1) (7).

Christiano et al. performed mutational analysis of a family with Bart's syndrome using DNA sequencing technique. The result showed the substitution of arginine in place of glycine in the triple helical domain of type VII collagen in Bart's syndrome (8).

The typical clinical feature of Bart's syndrome is a glistening, reddish color ulceration with extended, clearly defined borders usually seen on the foot (medial and dorsal aspects) and extending up to the shin. It may be unilateral or bilateral 
in position. This condition can be solitary or may have an association with other malformations or anomalies such as lowset abnormal ears, duodenal atresia, trachea-esophageal fistula, large fontanel, narrow palpebral fissures, micrognathia, cleft palate, hypoplasia of nose, joint contractures, rocker bottom feet, and genitourinary anomalies $(9,10)$.

Caksen et al. reported five newborn infants with ACC (one neonate had Adams-Oliver syndrome and another concomitant with Bart's syndrome) (11).

Narter et al. reported the first case report of a newborn infant with Bart's syndrome associated with pyloric and concomitant choanal atresia (12).

Casanova et al. reported a newborn with Bart's syndrome associated with the Herlitz form of lethal junctional epidermolysis bullosa on the basis of clinical features, cutaneous biopsy, and ultrastructural and immunohistochemical studies (13).

A recent clinical study by Chiaverini et al. concluded that ACC was a frequent manifestation in patients with dystrophic epidermolysis bullosa irrespective of the severity of the disease, and is due to leg rubbing in utero (14).

The majority of the dermatological lesions heal spontaneously with healing starting from the margins; after healing, the lesion remains as a smooth, yellowish, hairless, papery scar. Very rarely, hypertrophic scarring occurs, and these lesions, if linear, on the upper or lower limbs lead to joint contracture deformity. The prognosis is usually excellent if preventive measures are taken to prevent secondary bacterial infection and further trauma. Small residual lesions can be treated by removing the abnormal skin margins followed by primary closure. Larger scalp defects usually require early skin grafting or flap rotation (3).

Genetic counseling for this rare familial dermatological disorder is extremely important in families with affected members. DNAbased prenatal diagnosis using chorionic villus sampling or amniocentesis is available in the prenatal period (13).

\section{CONCLUSIONS}

ACC are a group of dermatological conditions characterized by the congenital absence of skin, usually on the vertex of the scalp.
They can occur as an isolated defect or can be associated with a number of other congenital anomalies. ACC with epidermolysis bullosa (Bart's syndrome) is a rare variant of aplasia cutis.

\section{REFERENCES}

1. Demmel U. Clinical aspects of congenital skin defects. I Congenital skin defects on the head of the newborn. Eur J Pediatr 1975; 121:21-50.

2. Dahmardehei M. A rare case of aplasia cutis congenita. Acta Med Iran 2013;51(5):341-4.

3. Frieden I. Aplasia cutis congenita: a clinical review and proposal for classification. J Am Acad Dermatol 1986; 14(4):646-60

4. Kruk Jeromin J, Jainik J, Rykala T. Aplasia Cutis Congenita of the scalp: Report of 16 cases. Dermatolo Surg 1998; 24 (5):549-53.

5. Bart BJ, Gorlin RJ, Anderson VE et al. Congenital localized absence of skin and associated abnormalities resembling epidermolysis bullosa: A new syndrome. Arch Dermatol 1966;93:296-303.

6. Kanzler MH, Smoller B, Woodley DT. Congenital localised absence of skin as a manifestation of epidermolysis bullosa. Arch Dermatol 1992; 128:1087-90.

7. Zelickson B, Matsumura K, Kist D et al. Bart's syndrome ultrastuctural and genetic linkage. Arch Dermatol 1995; 131:663-8.

8. Christiano AM, Bart BJ, Epstein EH et al. Genetic basis of Bart's syndrome: A glycine substitution mutation in type VII collagen gene. J Invest dermatol 1996; 106:778-80.

9. McCarthy MA, Clarke T, Powell FC. Epidermolysis bullosa and aplasia cutis. Int J Derm 1991; 30:481-484.

10. Carmi R, Sofer S, Karplus M. Aplasia cutis congenita in two sibs discordant for pyloric atresia. Am J Med Genetics 1982; 11: 319328.

11. Caksen H, Kurtoglu S. Our experience with aplasia cutis congenita. J Dermatol 2002; 29(6):376-9.

12. Narter $F$, Büyükbabani $N$, Yararlı $H$ et al. Bart's syndrome associated with pyloric and choanal atresia. Turk J Pediatr 2013;55(2):214-7.

13. Casanova JMI, Martí RM, Baradad M et al. Bart syndrome associated to lethal junctional epidermolysis bullosa (Herlitz form). Actas Dermosifiliogr 2006;97(10):658-61.

14. Chiaverini C, Charlesworth A, Fernandez A, Barbarot $S$, Bessis $D$, Bodemer $C$ et al. Aplasia cutis congenita with dystrophic epidermolysis bullosa: clinical and mutational study. Br J Dermatol 2014 Apr;170(4):901-6. doi: 10.1111/bjd.12741. PubMed PMID: 24252097. 